\title{
EFICÁCIA DOS MÉTODOS TERAPÊUTICOS NA APNEIA OBSTRUTIVA DO SONO EM PEDIATRIA: EVIDÊNCIAS CIENTÍFICAS
}

\section{ARTIGO DE REVISÃO}

MARTINS, Renata Renó ${ }^{1}$

MARTINS, Renan Renó

GONÇALVES, Maria Teresa Renó3

MARTINS, Renata Renó. GONÇALVES, Maria Teresa Renó. GONÇALVES, Maria Teresa Renó. Eficácia dos métodos terapêuticos na apneia obstrutiva do sono em pediatria: Evidências científicas. Revista Científica Multidisciplinar Núcleo do Conhecimento. Ano 04, Ed. 08, Vol. 07, pp. 48-63. Agosto de 2019. ISSN: 2448-0959

\section{RESUMO}

Objetivo: O estudo tem como objetivo elucidar o assunto, com base na literatura atualizada, descrevendo a eficácia dos métodos terapêuticos empregados na apneia obstrutiva do sono em pediatria. Metodologia: Trata-se de uma revisão sistemática da literatura. Foi utilizado o banco de dados online da PubMed. Os critérios de inclusão dos resumos para busca do texto completo foram: artigos publicados nos últimos 5 anos em inglês e com desenho de estudo epidemiológico estudo clínico randomizado. Foram excluídas as duplicidades, artigos com acesso restrito ao texto completo e

${ }^{1}$ Graduanda em Medicina pela Universidade José do Rosário Vellano (UNIFENAS) Alfenas, Brasil.

${ }^{2}$ Graduando em Medicina pela Faculdade da Amazônia (FAMAZ) - Belém, Brasil.

${ }^{3}$ Médica Especialista em Oftalmologia pelo Hospital dos Servidores - São Paulo, Brasil. Graduada em Medicina pela Faculdade de Medicina de Itajubá (FMIT) - Itajubá, Brasil. Professora do Curso de Medicina da Universidade Federal do Amapá (UNIFAP) - Macapá, Brasil. 
artigos cujo conteúdo não correspondia aos critérios definidos. Conclusões: Os benefícios da adenotonsilectomia já estão bem estabelecidos da literatura científica, porém outros métodos como o uso da cafeína, aparelhos de avanço mandibular e os dispositivos de pressão expiratória final nasal necessitam de mais estudos para comprovar um impacto positivo no tratamento da SAOS.

Descritores: Apneia Obstrutiva do Sono, terapêutica, pediatria.

\section{INTRODUÇÃO}

O ronco é decorrente da resistência das vias aéreas superiores, e a síndrome da apneia obstrutiva do sono (SAOS) é caracterizada por eventos de obstrução das vias aérea superiores (VAS) durante o sono. ${ }^{1}$

É estimado que a prevalência de ronco em crianças seja de 3 a 12\%; a de SAOS, de 0,7 a 3\%. A SAOS é predominante em pré-escolares, nesse período ocorre com mais frequência a hipertrofia do tecido linfoide nas VAS, porém pode se manifestar em qualquer idade. A SAOS é considerada uma questão de saúde pública emergente, estando associada a obesidade, hiperatividade e déficit de atenção. ${ }^{2}$

O distúrbio respiratório mais frequente associado ao sono é a apneia obstrutiva do sono. A ausência de conscientização entre a população e os recursos humanos da área da saúde faz com que cerca de $80-90 \%$ das pessoas com SAOS não tenham diagnóstico confirmado. Vários eventos cardiovasculares estão associados à SAOS não tratada, tais como fibrilação arterial, hipertensão arterial sistêmica, insuficiência cardíaca e acidente vascular encefálico. O paciente ainda pode apresentar excesso de sono no período diurno, qualidade de vida reduzida e risco para acidentes no trânsito. ${ }^{3}$

A SAOS na pediatria é uma disfunção da respiração que acontece no período do sono e é definido por longos períodos de obstrução das vias aéreas parcial e por eventos intermitentes de obstrução completa das vias aéreas superiores, o que prejudica na 
ventilação e o sono. No adulto o quadro ocorre de modo diferenciado quanto a apresentação clínica, fisiopatologia, diagnóstico e tratamento. ${ }^{4}$

O diagnóstico dos distúrbios respiratórios do sono e o encaminhamento para o tratamento têm como foco reduzir morbidades, principalmente distúrbios cardiológicos, metabólicos e cognitivos. ${ }^{5-8}$ A Academia Americana de Pediatria aconselha como padrão-ouro a realização de polissonografia (PSG) no período noturno para fechar o diagnóstico de SAOS em crianças com apresentações clínicas dos DRS..$^{9,10}$

Ultimamente a atenção tem sido focada na SAOS associada com más oclusões e más formações maxilo-faciais. As crianças com SAOS de nível mediano que estão em tratamento para distúrbios do sono associados a disfunções respiratórias apresentam obstruções das vias aéreas associadas a um septo nasal desviado com a maxila estreita. Muitas crianças com SAOS possuem alterações craniofaciais em maxila e mandíbula como também na estrutura do espaço respiratório. ${ }^{11}$

O tratamento da SAOS pode ser clínico (higiene do sono e pressão positiva nas vias aéreas) ou cirúrgico. ${ }^{12} \mathrm{~A}$ melhora no comportamento e atenção e possivelmente em habilidades cognitivas se deve como resultado do tratamento instituído para SAOS. ${ }^{13}$

O estudo tem como objetivo elucidar o assunto, com base na literatura atualizada, descrevendo a eficácia dos métodos terapêuticos empregados na apneia obstrutiva do sono em pediatria.

\section{METODOLOGIA}

Trata-se de uma revisão sistemática da literatura. Foi utilizado o banco de dados online da PubMed. Os critérios de inclusão dos resumos para busca do texto completo foram: artigos publicados nos últimos 5 anos (de 2014 a 2019) em inglês e com desenho de estudo epidemiológico (estudo clínico randomizado). Foram excluídas as duplicidades, artigos com acesso restrito ao texto completo e artigos cujo conteúdo não correspondia aos critérios definidos. 
$\mathrm{Na}$ estratégia de busca nas bases PubMed foram utilizadas palavras-chave definidas conforme sua descrição no MeSH (Medical Subject Headings), buscando-se: "child" OR "children" AND "obstructive sleep apnoea" OR "sleep apnea, obstructive" OR "sleep" AND "apnea" AND "obstructive" OR "obstructive sleep apnea" OR "obstructive" AND "sleep" AND "apnea" AND Randomized Controlled Trial AND "2014/05/26" : "2019/05/24" AND "humans".

Ao total obtiveram-se cerca de 35 documentos e selecionados 8 documentos de acordo com os critérios definidos.

Foi construída uma tabela para auxiliar na análise e na síntese dos artigos selecionados, sendo que contemplados os seguintes aspectos: nome dos autores e ano de publicação, amostragem, metodologia e resultados/conclusão.

\section{REFERENCIAL TEÓRICO}

\subsection{FISIOPATOLOGIA}

O sono reduz o estímulo respiratório alterando a função e o controle do sistema respiratório. Estas alterações modificam as vias aéreas superiores e a relação de troca de gases em crianças sadias e nas com patologias respiratórias ou de sistema nervoso. ${ }^{14}$ Geralmente o tônus muscular reduz durante o sono, é comum que crianças que apresentam sintomas à noite possam durante o dia não ter sintoma nenhum. ${ }^{15}$ Uma menor parcela delas, que possuem uma severa obstrução de VAS, apresenta ruídos na respiração e o aumento do esforço respiratório mesmo quando acordadas. ${ }^{14}$

$\mathrm{Na}$ SAOS em crianças, o tônus neuromotor faríngeo aumenta, em especial do músculo genioglosso, levando a um padrão de obstrução parcial e constante das vias aéreas superiores, além da hipercapnia e da hipóxia. Este padrão é estendido em tempo pois o despertar não ocorre. ${ }^{15}$ É mais comuns em as apneias cíclicas, com rompimento completo do fluxo aéreo e consequentes despertares, já nas crianças não é muito frequente. ${ }^{15,16}$ Os acordares em crianças são incomuns e quanto menor a idade mais raro se torna. ${ }^{15}$ 
Os despertares, devido às apneias, geram a recuperação a permeabilidade da faringe, porém mantém a base para a fragmentação do sono e o comprometimento neurocognitivo visualizados em crianças constantemente. ${ }^{17}$

\subsection{PRINCIPAIS FATORES ETIOLÓGICOS}

A SAOS tem etiologia multifatorial nas crianças e a ocorrência está relacionada a fatores obstrutivos estruturais e neuromotores. ${ }^{16}$

A hipertrofia das tonsilas faríngeas ou palatinas é colocado como a causa mais frequente em SAOS. ${ }^{18-20}$

Nas crianças a obesidade também é uma das razões mais comum de SAOS, devido o acúmulo de gordura na faringe e o aumento do volume abdominal e torácico ocorre um esforço respiratório. É preciso que disfunções neuromotores estejam presentes para que ocorra a SAOS, e a menor frequência de SAOS em crianças com peso ideal é uma grande evidência. ${ }^{14,21,22}$

\subsection{QUADRO CLÍNICO}

A maioria das crianças não possui sonolência excessiva diurna, mas algumas crianças têm sonolência. ${ }^{23}$ Outras encontram-se obesas; algumas crianças apresentam crescimento subótimo ou crescimento lentificado. Na SAOS grave algumas crianças apresentam tecido adenotonsilar reduzido, enquanto outras, com hipertrofia do mesmo, possuem quadros clínicos leves ou mesmo até assintomáticos..$^{23,24}$

O ronco e a dificuldade para respirar no sono são relatados com as queixas mais frequentes durante a infância; algumas crianças poderão apresentar um padrão respiratório ruidoso e difícil; respiração bucal e quadros de infecções do trato respiratório superior e de otite média frequentes. ${ }^{23-25}$ 


\subsection{DIAGNÓSTICO}

A avaliação adicional é definida após a realização da triagem por meio da história clínica e exame físico. ${ }^{26}$ Brouillette et al. ${ }^{27}$ evidenciaram uma sensibilidade e especificidade baixa em um score com história clínica e exame físico para diagnóstico de SAOS. O exame padrão-ouro para o diagnóstico de SAOS é a polissonografia em ambiente adequado. ${ }^{22,26,28,29}$

Através do quadro clínico o diagnóstico da SAOS deve ser suspeitado, mas a certeza diagnóstica é realizada por meio do exame polissonográfico. ${ }^{30-32}$

As alterações estruturais relacionadas podem ser estabelecidas por exames complementares. As alterações estruturais das VAS podem ser avaliadas pelo exame de nasofaringolaringoscopia. ${ }^{33}$

Mais recentemente, Gozal et al. ${ }^{34}$, evidenciaram proteínas urinárias uromodulina, orosomucoide-1, calicreína e urocortina-3 como possíveis marcadores para distinguir o ronco primário da SAOS.

Atualmente são descritas novas tecnologias para a identificação do fracionamento do sono e assim realizar o diagnóstico diferencial entre ronco primário, síndrome da resistência da VAS e SAOS. São exemplos dessas tecnologias os marcadores de atividade simpática como a tonometria de artéria periférica e o tempo de trânsito de pulso, que detectam eventos com despertares relacionados a distúrbios respiratórios. ${ }^{35}$

\subsection{CONSEQUÊNCIAS}

As consequências mais comuns da SAOS em crianças são: hipertensão pulmonar, que pode gerar à insuficiência cardíaca e ao cor pulmonale, desenvolvimento pôndero-estatural reduzido, alterações comportamentais, redução na concentração, hipersonolência diurna e aprendizado na escola prejudicado. ${ }^{19,36}$ 
É essencial que a SAOS seja diagnóstica e tratada o mais rápido possível para tentar impedir a instalação das consequências, devido sua atuação deletéria no desenvolvimento da criança. ${ }^{20,37}$

\subsection{TRATAMENTO}

A terapia a ser adotada será guiada pela etiologia subjacente, gravidade, história natural e opções terapêuticas que proporcione uma melhora na permeabilidade da VAS. A adenotonsilectomia é o plano terapêutico de $1^{a}$ linha quando possui hipertrofia adenotonsilar. .,38 $^{4}$

Para o tratamento da SAOS em pediatria associado com hipertrofia de tonsilas, o mais adequado é a adenotonsilectomia ${ }^{16,26,29}$

O CPAP (Pressão Positiva Contínua nas Vias Aéreas) está indicado quando a adenotonsilectomia não é indicada, naqueles em que a cirurgia não reduziu os sintomas e na malformação craniana. ${ }^{16,21,26}$

O corticosteróide tópico utilizado por no mínimo 6 semanas é o único tratamento medicamentoso que reduz os sintomas da SAOS e os índices no exame de polissonografia em crianças. ${ }^{39}$

A traqueostomia é realizada para alívio na obstrução das vias aéreas superiores quando outros planos terapêuticos tenham falhado para tratar a SAOS moderada a grave ou enquanto espera a realização da cirurgia craniofacial. ${ }^{40}$

É preciso se atentar ao tratamento das condições médicas subjacentes, como a redução de peso nas crianças com sobrepeso e obesas e o tratamento de refluxo gastroesofágico, rinite, distúrbios da tireoide e asma, pois o tratamento dessas situações impacta positivamente no tratamento da SAOS. ${ }^{41}$ 


\section{RESULTADOS E DISCUSSÃO}

Foram incluídos 8 artigos de acordo com os critérios de inclusão e exclusão (Tabela 1). O total de crianças abrangidas na pesquisa foi de 1747.

Tabela 1. Artigos incluídos na revisão de acordo com autor, ano de publicação, amostragem, metodologia e conclusão.

\begin{tabular}{|c|c|c|c|}
\hline $\begin{array}{l}\text { Autoria/Ano } \\
\text { de } \\
\text { publicação }\end{array}$ & Amostra & Metodologia & Conclusão \\
\hline $\begin{array}{l}\text { Yang et al. } \\
(2017)^{42}\end{array}$ & 183 & $\begin{array}{l}\text { Ensaio Clínico } \\
\text { Randomizado }\end{array}$ & $\begin{array}{l}\text { O tratamento combinado de } \\
\text { Montelucaste de Sódio via oral e } \\
\text { Furoato de Mometasona via intranasal } \\
\text { é superior do que o uso isolado de } \\
\text { qualquer uma das medicações. }\end{array}$ \\
\hline $\begin{array}{l}\text { Borgstrom et } \\
\text { al. } \\
(2017)^{43}\end{array}$ & 79 & $\begin{array}{l}\text { Ensaio Clínico } \\
\text { Randomizado }\end{array}$ & $\begin{array}{l}\text { Os resultados sugerem que a } \\
\text { adenotonsilotomia não é inferior à } \\
\text { adenotonsilectomia no tratamento da } \\
\text { SAOS pediátrica em relação aos } \\
\text { resultados da polissonografia } \\
\text { realizada após } 1 \text { ano. }\end{array}$ \\
\hline $\begin{array}{l}\text { Xiao et al. } \\
(2017)^{44}\end{array}$ & 353 & $\begin{array}{l}\text { Ensaio Clínico } \\
\text { Randomizado }\end{array}$ & $\begin{array}{l}\text { A adenotonsilectomia foi eficaz na } \\
\text { redução dos eventos respiratórios } \\
\text { francos em crianças com SAOS e na } \\
\text { assincronia toracoabdominal durante } \\
\text { o sono tranquilo. }\end{array}$ \\
\hline $\begin{array}{l}\text { Paruthi et al. } \\
(2016)^{45}\end{array}$ & 464 & $\begin{array}{l}\text { Ensaio Clínico } \\
\text { Randomizado }\end{array}$ & $\begin{array}{l}\text { Foi observada uma melhora na } \\
\text { sonolência maior após } \\
\text { adenotonsilectomia precoce do que } \\
\text { os cuidados de suporte. }\end{array}$ \\
\hline
\end{tabular}




\begin{tabular}{|c|c|c|c|}
\hline $\begin{array}{l}\text { Machado- } \\
\text { Júnior et al. } \\
(2016)^{46}\end{array}$ & 14 & $\begin{array}{l}\text { Ensaio Clínico } \\
\text { Randomizado }\end{array}$ & $\begin{array}{l}\text { Houve uma diminuição no índice de } \\
\text { apneia-hipopneia um ano após a } \\
\text { implementação do uso de dispositivos } \\
\text { de avanço mandibular, em } \\
\text { comparação com o grupo que não } \\
\text { utilizou esses dispositivos. }\end{array}$ \\
\hline $\begin{array}{l}\text { Garetz et al. } \\
(2015)^{47}\end{array}$ & 453 & $\begin{array}{l}\text { Ensaio Clínico } \\
\text { Randomizado }\end{array}$ & $\begin{array}{l}\text { A adenotonsilectomia comparada } \\
\text { com a espera vigilante resultou em } \\
\text { melhorias significativamente da } \\
\text { qualidade de vida e nos sintomas da } \\
\text { SAOS. }\end{array}$ \\
\hline $\begin{array}{l}\text { Marcus et al. } \\
(2014)^{48}\end{array}$ & 201 & $\begin{array}{l}\text { Ensaio Clínico } \\
\text { Randomizado }\end{array}$ & $\begin{array}{l}\text { Administração de cafeína neonatal } \\
\text { terapêutico não tem efeitos a longo } \\
\text { prazo sobre a duração do sono } \\
\text { ou apneia do sono durante a infância. }\end{array}$ \\
\hline $\begin{array}{l}\text { Kureshi et al. } \\
(2014)^{49}\end{array}$ & 14 & $\begin{array}{l}\text { Ensaio Clínico } \\
\text { Randomizado }\end{array}$ & $\begin{array}{l}\text { Os dispositivos de Pressão } \\
\text { Expiratória Final Nasal são uma } \\
\text { terapia alternativa potencial para a } \\
\text { SAOS em um pequeno subgrupo } \\
\text { de crianças. }\end{array}$ \\
\hline
\end{tabular}

Fonte: (Próprio autor)

No estudo de Yang et al. ${ }^{42}$ revelou que após 12 semanas de tratamento com Montelucaste de Sódio (MS) via oral ou Furoato de Mometasona (FM) via intranasal, os distúrbios respiratórios em parte das crianças com SAOS leve poderiam ser aliviados, e a taxa efetiva era de $62,4 \%$ e $63,1 \%$, respectivamente. A diferença entre estes dois tratamentos não foi estatisticamente significativa. Após 12 semanas de tratamento combinado de MS (antagonista do receptor de leucotrienos) e FM (corticoide), a taxa efetiva $(73,7 \%)$ foi significativamente maior do que a do tratamento com um único medicamento. 
O plano terapêutico é composto de corticosteroides nasais e de drogas antagonistas de receptores de leucotrienos ou a associação das mesmas, com resultado efetivo na SAOS residual após a adenotonsilectomia. ${ }^{25,38}$ Kheirandish-Gozal et al. ${ }^{50}$, em 2008, evidenciaram que o grupo tratado com budesonida nasal obteve uma redução no índice de apneia e hipopneia (IAH) e o efeito dos corticosteroides intranasais permaneceram por no mínimo 8 semanas após o uso descontinuado do mesmo. Em outro estudo com crianças com SAOS leve que receberam medicação montelucaste por 16 semanas, evidenciou-se uma diminuição do tecido adenotonsilar e do IAH. ${ }^{51}$

No estudo de Marcus et al. ${ }^{48}$, o uso de cafeína neonatal para a apneia da prematuridade mostrou diminuir a morbidade em bebês prematuros e, portanto, é amplamente utilizado em unidades de terapia intensiva neonatal. Este estudo mostrou que a administração terapêutica de cafeína neonatal não tem efeitos a longo prazo na qualidade ou quantidade do sono, ou em condições patológicas durante o sono, como a SAOS. 48

Inúmeros estudos têm relatado os efeitos positivos da cafeína no tratamento da apneia no recém-nascido prematuro, pois reduz os episódios de apneia, uso ventilação mecânica nos prematuros, da pressão arterial de $\mathrm{CO} 2$, da concentração de íons de hidrogênio, acarretando em um padrão respiratório adequado e aumentando a ventilação alveolar. ${ }^{52,53}$

O estudo de Borgstrom et al. ${ }^{43}$ foi o primeiro a comparar a adenotonsilotomia (ATT) e adenotonsilectomia (ATE) em SAOS pediátrica através da PSG. Os resultados da PSG sugeriram que a ATT não é inferior a ATE no tratamento da SAOS pediátrica. No entanto, cinco pacientes (idade média de 32 meses) no grupo ATT necessitaram realizar ATE e, portanto, devem ser considerados como fracasso da ATT.

Outro estudo não randomizado de ATE versus ATT, que incluiu 29 crianças, mostrou melhora semelhante após 6 meses em ambos os grupos, com reduções médias do IAH comparáveis: $96 \%$ no grupo ATT e $95 \%$ no grupo ATE. ${ }^{54}$ 
Um recente estudo sueco baseado em> 28.000 crianças relatou um risco 7 vezes maior de reoperação após ATT do que após ATE, com a diferença mais marcante entre as crianças mais novas. ${ }^{55}$

O principal achado da pesquisa Xiao et al. ${ }^{44}$ é a redução da assincronia toracoabdominal durante o sono tranquilo e livre de eventos em crianças com SAOS após a ATT, indicando uma redução geral no esforço inspiratório.

No estudo controlado randomizado de adenoamigdalectomia para o tratamento da SAOS pediátrica, foi observado uma melhora significativa nos relatos dos pais sobre a sonolência em crianças submetidas à adenotonsilectomia em comparação àquelas que receberam cuidado de apoio. ${ }^{45}$

Um grande estudo multicêntrico, prospectivo, randomizado e controlado de adenotonsilectomia para SAOS pediátrica documentado por PSG constatou que os sintomas chave e a qualidade de vida melhoraram substancialmente após a ATE do que o tratamento com cuidados de suporte. ${ }^{47}$

O estudo de Kureshi et al. ${ }^{49}$ mostrou que algumas crianças respondem ao nEPAP (Pressão Expiratória Final Nasal) , mas existe uma variabilidade individual na resposta. Algumas crianças tiveram uma resposta clinicamente significativa. Os dispositivos nEPAP podem ser uma terapia alternativa potencial para a SAOS para um pequeno subconjunto de crianças com SAOS persistente após a cirurgia ou aquelas que não são capazes de tolerar o CPAP. 49

Vários pesquisadores, em diferentes países, estudam outras alternativas de tratamento da SAOS, em especial um dispositivo denominado Provent. O Provent nasal ou nEPAP é um adesivo descartável utilizado em ambas as narinas externamente. Cada adesivo contém uma válvula que oferece uma resistência expiratória de $80 \mathrm{cmH} 20$ durante a expiração (embora existam válvulas com menores resistências, esta parece oferecer maior benefício no que se refere à correção dos eventos respiratórios). ${ }^{56}$ 
Os resultados do estudo de Machado-Júnior et al. demonstram que houve diminuição do IAH após o uso dos dispositivos de avanço mandibular e aumento desse índice no grupo controle. ${ }^{46}$

As atuais evidências limitadas podem ser sugestivas de que os aparelhos de avanço mandibular (AAM) resultam em melhorias nos escores do IAH, mas não é possível concluir que os AAM sejam eficazes no tratamento da SAOS pediátrica. ${ }^{57,58}$

\section{CONCLUSÃO}

Os benefícios da adenotonsilectomia já estão bem estabelecidos da literatura científica, porém outros métodos como o uso da cafeína, aparelhos de avanço mandibular e os dispositivos de pressão expiratória final nasal necessitam de mais estudos para comprovar um impacto positivo no tratamento da SAOS.

\section{REFERÊNCIAS}

1. Franklin $\mathrm{K}$, et al. Effects and Side-Effects of Surgery for Snoring and Obstructive Sleep Apnea - A Systematic Review. Sleep Medicine Reviews, Suiça, n. , p.2736, 10 jan. 2009.

2. Gregório PB, et al. Sintomas da síndrome de apnéia-hipopnéia obstrutiva do sono em crianças. Jornal Brasileiro de Pneumologia, São Paulo, n.6, 01 jun. 2008.

3. Ahrens A, Mcgrath C, Hägg U. A systematic review of the efficacy of oral appliance design in the management of obstructive sleep apnoea. European Journal Of Orthodontics, Hong Kong, p.318- 324, 13 jan. 2011.

4. Schechter MS. Section on Pediatric Pulmonology, Subcommittee on Obstructive Sleep Apnea Syndrome. Technical report: diagnosis and management of childhood obstructive sleep apnea syndrome. Pediatrics. 2002;109(4):e69. 
5. Amin RS, Kimball TR, Kalra M, Jeffries JL, Carroll JL, Bean JA, et al. Left ventricular function in children with sleep-disordered breathing. Am J Cardiol. 2005;95:801---4.

6. Hunter SJ, Gozal D, Smith DL, Philby MF, Kaylegian J, Kheirandish-Gozal L. Effect of sleep-disordered breathing severity on cognitive performance measures in a large community cohort of young school-aged children. Am J Respir Crit Care Med. 2016;194:739---47.

7. Koren D, Gozal D, Bhattacharjee R, Philby MF, KheirandishGozal L. Impact of adenotonsillectomy on insulin resistance and lipoprotein profile in nonobese and obese children. Chest. 2016;149:999---1010.

8. Pires PJ, Mattiello R, Lumertz MS, Morsch TP, Fagondes SC, Nunes ML, et al. Validation of the Brazilian version of the Pediatric Obstructive Sleep Apnea Screening Tool questionnaire. J Pediatr (Rio J). 2019;95:231---7.

9. Wise M, Nichols C, Grigg-Damberger M, Marcus C, Witmans M, Kirk V, et al. Respiratory indications for polysomnography in children: an evidence-based review. Sleep. 2011;34:389---98.

10. Kheirandish-Gozal L, Bandla HP, Gozal D. Montelukast for children with obstructive sleep apnea: results of a double-blind, randomized, placebocontrolled trial. Ann Am Thorac Soc. 2016;13:1736---41.

11. Pirelli $P$, et al. Orthodontics and Obstructive Sleep Apnea in Children. Med Clin N Am, Roma, p.517-539, 2010.

12. Balbani APS, Weber SAT, Montovani JC. Atualização em síndrome da apnéia obstrutiva do sono na infância. Rev. Bras. Otorrinolaringol. 2005, vol.71, n.1, pp. 74-80.

13. Pediatrics, American Academy Of et al. Diagnosis and Management of Childhood Obstructive Sleep Apnea Syndrome. Pediatrics, Illinois, p.714- 755, 01 set. 2012.

14. American Thoracic Society. Standards and Indications for Cardiopulmonary Sleep Studies in Children. Am J Respir Crit Care Med 1996; 153: 866-78.

15. Marcus CL. Pathophysiology of childhood obstructive sleep apnea: current concepts. Resp Physiol 2000; 119: 143-54. 
16. Ward SL, Marcus CL. Obstructive sleep apnea in infants and young children. J Clin Neurophysiol 1996; 13: 198-207.

17. Bourke RS, Anderson V, Yang JS, Jackman AR, Killedar A, Nixon GM et al. Neurobehavioral function is impaired in children with all severities of sleep disordered breathing. Sleep Med. 2011;12:222-9.

18. Sleep-related breathing disorders in adults: recommendations for syndrome de \pm nitions and measurement techniques in clinical research. The Report of an American Academy of Sleep Medicine Task Force. Sleep. 1999; 22(5):667-89

19. American Thoracic Society. Indications and standards for use of nasal continuous positive airway pressure (CPAP) in sleep apnea syndromes. Am J Respir Crit Care Med. 1994; 150(6Pt1):1738-45.

20. Di Francesco RC, Fortes FSG, Komatsu CL. Melhora da qualidade de vida em crianças após adenoamigdalectomia. Rev. Bras. Otorrinolaringol. 2004 novdez; 70(6):748-51.

21. Lipton AJ, Gozal D. Treatment of obstruction sleep apnea in children. Do we really know how? Sleep Med Rev 2003; 7: 61-80.

22. American Thoracic Society. Cardiorespiratory sleep studies in children. Am J Respir Crit Care Med 1999; 160: 1381-7.

23. Katz ES, D'Ambrosio CM. Pathophysiology of Pediatric Obstructive Sleep Apnea. Proc Am Thorac Soc.2008;5:253-262.

24. Dayyat E, Kheirandish-Gozal, Gozal D. Childhood Obstructive Sleep Apnea: One or Two Distinct Disease Entities? Sleep Med Clin. 2007;2:433-44.

25. Katz ES, D’Ambrosio CM. Pediatric Obstructive Sleep Apnea Syndrome. Clin Chest Med. 2010;31:221-234.

26. American Academy of Pediatrics. Clinical practice guideline diagnosis and management of childhood obstructive sleep apnea syndrome. Pediatrics 2002; 109: 704-12.

27. Brouillette RT, Morielle A, Leimanis A, Waters KA, Luciano R, Ducharme FM. Nocturnal pulse oximetry as an abbreviated testing modality for pediatric obstructive sleep apnea. Pediatrcis 2000; 105: 405-12. 
28. Guilleminault C, Eldridge FL, Simmons FB, Dement WC. Sleep apnea in eight children. Pediatrics 1976; 58: 28-32.

29. Nieminen $P$, Tolonen $U$, Löppönen $H$. Snoring and obstructive sep apnea in children - a 6 month follow-up study. Arch Otolaryngol Head Neck Surg 2000; 126: 481-6.

30. Kryger MH, Roth T, Dement WC. Principles and practice of sleep medicine. 4th ed. Philadelphia: Elsevier/Saunders; c2005.

31. Martinez D. Insônia na prática clínica. Porto Alegre: Artmed; 2005.

32. Tufik S. Medicina e biologia do sono. São Paulo: Manole; 2008.

33. Muzumdar $\mathrm{H}$, Arens $\mathrm{R}$. Diagnostic issues in pediatric obstructive sleep apnea. Proc Am Thorac Soc. 2008;5:263-73.

34. Gozal D, Jortani S, Snow AB, Kheirandish-Gozal L, Bhattacharjee R, Kim J, et al. Two-dimensional differential in-gel electrophoresis proteomic approaches reveal urine candidate biomarkers in pediatric obstructive sleep apnea. Am J Respir Crit Care Med. 2009;180:1253- 6.

35. Friedman NR. Novel methods to diagnose obstructive sleep apnea in children. Operative Techniques in Otolaryngology- Head and Neck Surgery. 2012; 23:79-83.

36. Skinner MA, Kingshott RN, Jones DR, Taylor DR. Lack of efficacy for a cervicomandibular support collar in the management of obstructive sleep apnea. Chest. 2004; 125(1):118-26.

37. Valera FCP, Demarco RC, Anselmo-Lima WT. Síndrome da apnéia e da hipopnéia obstrutivas do sono (SAHOS) em crianças. Rev. Bras. Otorrinolaringol. 2004; 70(2):232-7.

38. Marcus CL, Brooks LJ, Draper KA, Gozal D, Halbower AC, Jones J et al. Diagnosis and Management of Childhood Obstructive Sleep Apnea Syndrome. American Academy of Pediatrics. Pediatrics. 2012;130:576-84.

39. Brouillette RT, Manoukian JJ, Ducharme FM, Oudiane K, Earle LG, Ladan S. Morielli A. Efficacy of fluticasone nasal spray for pediatric obstructive sleep apnea. J Pediatr 2001; 138: 838-44. 
40. Kaditis A, Kheirandish-Gozal L, Gozal D. Algorithm for the diagnosis and treatment of pediatric OSA: A proposal of two pediatric sleep centers. Sleep Medicine. 2012; 13:217-27.

41. Ann C. Halbowera, Brian M. McGinleyb and Philip L. Smith. Treatment alternatives for sleep-disordered breathing in the pediatric population. Curr Opin Pulm Med. 2008,14:551-8.

42. Yang DZ, Liang J, Zhang F, Yao HB, Shu Y. Clinical effect of montelukast sodium combined with inhaled corticosteroids in the treatment of OSAS children. Medicine (Baltimore). 96(19):e6628.

doi: 10.1097/MD.0000000000006628. Epub 2017 May 12. PubMed PMID: 28489737; PubMed Central PMCID: PMC5428571.

43. Borgstrom A, Nerfeldt P, Friberg D. Adenotonsillotomy versus adenotonsillectomy in pediatric obstructive sleep apnea: an RCT. Pediatrics. 2017;139(4):e20163314. doi: 10.1542/peds.2016-3314.

44. Xiao L, Sarah I, Yvonne P, Declan K, James M, Mathias B. Adenotonsillectomy for childhood obstructive sleep apnoea reduces thoraco-abdominal asynchrony but spontaneous apnoea-hypopnoea index normalisation does not. European Respiratory Journal Jan 2017, 49 (1) 1601177; DOI: 10.1183 / 13993003.01177-2016

45. Paruthi S, Buchanan P, Weng J, et al. Effect of Adenotonsillectomy on ParentReported Sleepiness in Children with Obstructive Sleep Apnea. Sleep. 2016;39(11):2005-2012. Published 2016 Nov 1. doi:10.5665/sleep.6232

46. Machado-Júnior AJ, Signorelli LG, Zancanella E, Crespo AN. Randomized controlled study of a mandibular advancement appliance for the treatment of obstructive sleep apnea in children: A pilot study. Med Oral Patol Oral Cir Bucal. 2016;21(4):e403-e407. Published 2016 Mar 6. doi:10.4317/medoral.21072

47. Garetz SL, Mitchell RB, Parker PD, et al. Quality of life and obstructive sleep apnea symptoms after pediatric adenotonsillectomy. Pediatrics. 2015;135(2):e477-e486. doi:10.1542/peds.2014-0620. 
48. Marcus CL, Meltzer LJ, Roberts RS, et al. Long-term effects of caffeine therapy for apnea of prematurity on sleep at school age. Am J Respir Crit Care Med . 2014; 190 (7): 791-799. doi: 10.1164 / rccm.201406-10

49. Kureshi SA, Gallagher PR, McDonough JM, et al. Pilot study of nasal expiratory positive airway pressure devices for the treatment of childhood obstructive sleep apnea syndrome. J Clin Sleep Med . 2014; 10 (6): 663-669. Published 2014 de 15 de junho. Doi: 10.5664 / jcsm.3796 920C.

50. Kheirandish-Gozal L, Gozal D. Intranasal budesonide treatment for children with mild obstructive sleep apnea syndrome. Pediatrics. 2008;122:e149-55.

51. Goldbart AD, Goldman JL, Veling MC, Gozal D. Leukotriene modifier therapy for mild sleep-disordered breathing in children. Am J Respir Crit Care Med. 2005; 172:364-370.

52. Hentges CR, Guedes RR, Silveira RC, PRocianoy RS. Serum Levels of caffeine in umbilical cord and apnea of prematurity. $J$ Pediatr, v.86, n.2, p.137-142, 2010.

53. Pacifici GM. Clinical pharmacology of caffeine citrate in preterm infants. Medical Express, v.1, n.5, p.243-250, 2014.

54. Cantarella G, Viglione $S$,Forti $S$,Minetti A ,Pignataro L.Comparando a qualidade de vida pós-operatória em crianças após tonsilectomia intracapsular e amigdalectomia microdebridativa Auris Nasus Larynx . 2012 ; 39 ( 4 ): 407 410 pmid: 22118950

55.Odhagen E ,Sunnergren $O$,Hemlin C , et al. Risco de reoperação após tonsilotomia versus tonsilectomia: um estudo de coorte de base populacional. Eur Arch Otorhinolaryngol . 2016 . 273 ( 10 ): 3263 - 3268.

56. Garcia-Borreguero D, Larosa O, Bravo M. Parkinson's disease and sleep. Sleep Med Rev. 2003; 7:115-129.

57. Carvalho FR, Lentini-Oliveira D, Machado MA, Prado GF, Prado LB, Saconato $\mathrm{H}$. Oral appliances and functional orthopaedic appliances for obstructive sleep apnoea in children. Cochrane Database Syst Rev. 2007;2:CD005520. 
58. Nazarali N, Altalibi M, Nazarali S, Major MP, Flores-Mir C, Major PW. Mandibular advancement appliances for the treatment of paediatric obstructive sleep apnea: a systematic review. Eur J Orthod. 2015;37:618-26.

Enviado: Julho, 2019.

Aprovado: Agosto, 2019. 